\title{
LncRNA XIST promotes inflammation by downregulating GR $\alpha$ expression in the adenoids of children with OSAHS
}

\author{
ZHEN ZHOU $^{1,2}$, HAIFENG NI ${ }^{1}$, YONG LI ${ }^{1}$ and BO JIANG ${ }^{1}$ \\ ${ }^{1}$ Department of Otolaryngology, Head and Neck Surgery, Affiliated Hangzhou First People's Hospital, \\ Zhejiang University School of Medicine, Hangzhou, Zhejiang 310006; ${ }^{2}$ Department of Otolaryngology, \\ Head and Neck Surgery, Hangzhou Cancer Hospital, Hangzhou, Zhejiang 310002, P.R. China
}

Received February 20, 2020; Accepted July 31, 2020

DOI: $10.3892 / \mathrm{etm} .2021 .9931$

\begin{abstract}
Whether glucocorticoid receptor $\alpha(\mathrm{GR} \alpha)$ serves a role in obstructive sleep apnea/hypopnea syndrome (OSAHS) remains unclear. However, it has been reported that GR $\alpha$ expression is decreased in the adenoids of patients with OSAHS. The present study aimed to evaluate the role of GR $\alpha$ in OSAHS and the underlying mechanism. Bioinformatics assays revealed that long noncoding RNA (lncRNA) $\mathrm{X}$ inactivate-specific transcript (XIST) was closely associated with GR $\alpha$. Furthermore, reverse transcription-quantitative PCR showed that the expression of IncRNA XIST was significantly increased in the adenoids of patients with OSAHS compared with healthy controls. Further in vitro studies by Pearson correlation analysis, RNA pull-down assay, western blot analysis and ELISA demonstrated that XIST significantly decreased the expression of GR $\alpha$ and that significantly increased the production of inflammatory cytokines, including interleukin (IL)-8, tumor necrosis factor $\alpha$, IL-6 and IL-1 $\beta$, while the overexpression of GR $\alpha$ significantly decreased the production of these inflammatory cytokines in NP69 cells, a human nasopharyngeal epithelial cell line. Furthermore, XIST significantly increased the protein levels of nuclear factor $\kappa$-light-chain-enhancer of activated B cells (NF- $\mathrm{BB}$ ) subunits, including Rel-B, c-Rel, P52, P50 and P65, which are associated with the transcription of cytokines. The stimulatory effect of XIST was significantly inhibited by the NF- $\mathrm{KB}$ inhibitor EVP4593. These results indicated that the stimulatory effect of XIST was dependent on NF- $\kappa B$. In summary, the present study demonstrated that the XIST-GR $\alpha-N F-\kappa B$ signaling pathway
\end{abstract}

Correspondence to: Dr Haifeng Ni, Department of Otolaryngology, Head and Neck Surgery, Affiliated Hangzhou First People's Hospital, Zhejiang University School of Medicine, 261 Huan Sha Road, Hangzhou, Zhejiang 310006, P.R. China

E-mail: nihaifeng138@163.com

Key words: $\mathrm{X}$ inactivate-specific transcript, glucocorticoid receptor $\alpha$, nuclear factor $\kappa$-light-chain-enhanced of activated $\mathrm{B}$ cells, obstructive sleep apnea/hypopnea syndrome, inflammation contributed to inflammation in the adenoids of patients with OSAHS.

\section{Introduction}

Obstructive sleep apnea/hypopnea syndrome (OSAHS) is the most common type of apnea (1). It is characterized by recurrent episodes of partial or complete upper airway collapse during sleep (2). OSAHS has been demonstrated to be associated with several comorbidities, including cardiovascular and neurodegenerative disorders, such as Alzheimer's disease and Parkinson's disease (3-5). Previous findings have shown that the physiological and pharmacological actions of glucocorticoids are mediated by the glucocorticoid receptor (GR), which is a member of the nuclear receptor superfamily of ligand-dependent transcription factors (6). Following glucocorticoid binding, GR induces or represses the transcription of target genes, which comprise up to $10-20 \%$ of the human genome (7). For instance, GR has been previously reported to positively regulate the transcription of FNDC5 in the liver (8), whilst another study revealed that Angptl4 is a GR primary target gene in hepatocytes and adipocytes (9). The expression of GR $\alpha$ has been demonstrated to be decreased in patients with OSAHS. For instance, the expression of GR $\alpha$ in the tonsil tissues of children with OSAHS was significantly lower compared with the tonsil tissues of children without OSAHS (10). However, the mechanism by which GR $\alpha$ is dysregulated in patients with OSAHS remains unknown.

Long noncoding RNAs (IncRNAs) are a class of transcripts $>200$ nucleotides in length (11). Generally, lncRNAs do not encode proteins (12). Accumulating data have indicated that lncRNAs exert crucial functions in gene regulation, biological processes and several diseases, including inflammation (13) and immune responses (14). LncRNA X inactivate-specific transcript (XIST) is a 17-kb long RNA and is transcribed from the inactive $\mathrm{X}$ chromosome in female mammalians. Previous studies have indicated that the expression of XIST is upregulated in various diseases, such as in basal-like human breast cancer (15) and cystic fibrosis (16). However, it remains unclear whether XIST serves a role in OSAHS.

It has been well documented that nuclear factor $\kappa$-light-chain-enhancer of activated B cells (NF- $\kappa \mathrm{B})$ serves a vital role in inflammation (17). The IкB kinase enzyme 
complex is part of the upstream NF- $\mathrm{\kappa B}$ signal transduction cascade and the I $\kappa \mathrm{B} \alpha$ protein inactivates transcription factor of NF- $\kappa \mathrm{B}$ by inhibiting the nuclear localization signals of $\mathrm{NF}-\mathrm{KB}$ and sequestering it to an inactive state in the cytoplasm (18). NF- $\mathrm{kB}$ is a protein complex composed of homo- or heterodimers formed by different combinations of the five monomeric NF- $\kappa$ B subunits: p65/Rel A, Rel B, c-Rel, p50 and p52 (19). Activated NF- $\kappa B$ regulated cell proliferation, apoptosis and the production of inflammatory cytokines, including tumor necrosis factor (TNF)- $\alpha$, interleukin (IL)- $1 \beta$, IL-6 and IL-8 $(20,21)$.

In conclusion, the present study specially focused on the role and mechanism of GR $\alpha$ in adenoids of patients with OSAHS, the results of which may provide a potential novel insight for treatment of OSAHS.

\section{Materials and methods}

Patients and sample collection. The present study was approved by the Ethics Committee and Animal Care Committee of the Affiliated Hangzhou First People's Hospital, Zhejiang University School of Medicine, Hangzhou, China. The legal guardians of all the participants read and signed informed consent forms. Both inclusion and exclusion criteria were first assessed through review of the electronic medical record. Inclusion criteria were: i) Age $\leq 12$ years old; ii) able to obtain written informed consent from legal guardians; iii) hospitalized patients having a documented history of congestive heart failure in agreement with the 2013 ACCF/AHA heart failure definition (22); iv) anticipated hospitalization of $>24 \mathrm{~h}$. Exclusion criteria were: i) Established obstructive sleep apnea and/or previous exposure to positive airway pressure (PAP) therapy; ii) Presence of any active conditions that the investigators felt would interfere with testing or potential therapy (hemodynamic instability, respiratory failure, unconsciousness, pneumothorax, penetrating chest trauma, general nausea/vomiting, facial anomalies/facial trauma, upper gastrointestinal bleed or history of recent gastric surgery); iii) presence of a clinically significant illness or medical condition that the investigators felt would prohibit the subject from participating in the study (a number of subjects were ruled due to advanced COPD, malignancy or having a high baseline home oxygen requirement).

According to the apnea-hypopnea index, the patients were diagnosed with OSAHS when apnea-hypopnea occurred $\geq$ one time per hour, as previously described (23). Adenoid tissue specimens were collected from children with OSAHS $(n=26)$ and healthy donors $(\mathrm{n}=21)$ with myocarditis at the Department of Otorhinolaryngology, Affiliated Hangzhou First People's Hospital, Zhejiang University School of Medicine, from May 2017 to May 2018. Mean age of the OSAHS group (19 females and 7 males) was $6.88 \pm 3.18$ years. Mean age of the healthy donor group (19 females and 2 males) was $7.2 \pm 2.63$ years. No local or systemic glucocorticoid treatment was given to any of the patients within 2 weeks to 2 months prior to the operations.

Cell cultures. Human nasopharyngeal epithelial cell line NP69 purchased from American Type Culture Collection (ATCC) was cultured in keratinocyte-SFM medium (Invitrogen; Thermo Fisher Scientific, Inc.). All cells were grown in a humidified incubator with a $5 \% \mathrm{CO}_{2}$ atmosphere at $37^{\circ} \mathrm{C}$, as previously described (24). Additionally, EVP4593 (cat. no. HY-13812; MedChemExpress), an inhibitor of NF- $\mathrm{\kappa B}$ signaling, was used to treat NP69 cells. DMSO was used as the control.

Cell transfections. Total RNA was extracted from adenoid tissue tissues and NP69 cells using TRIzol ${ }^{\circledR}$ reagent (Invitrogen; Thermo Fisher Scientific, Inc.) and synthesized into first-strand cDNA using an M-MLV Reverse Transcriptase kit (cat. no. 28025013; Invitrogen; Thermo Fisher Scientific, Inc.) as previously described (25). Full-length XIST was amplified from female genomic DNA (Roche diagnostics) and then digested with $X b a \mathrm{I}$ to obtain nucleotides 105-10747 of the XIST cDNA sequence (accession no. M97168) and inserted into pcDNA3.1 vectors (Invitrogen; Thermo Fisher Scientific, Inc.) to overexpress XIST. GR $\alpha$ overexpression plasmids were constructed using the pcDNA3.1 backbone from Sangon Biotechnology Co., Ltd. XIST short hairpin (sh) RNA sequences were inserted into shRNA vectors (Shanghai GenePharma Co., Ltd.). The actual DNA sequence of the shRNA that was ligated into the pGPH1 vector were as follows: sh-NC, 5'-CACCGCTATGATATCGTCTGTTTCAAGAGA ACAGACGATATCATAGCTTTTTTG-3' and sh-XIST, 5'-CACCGCATCTGACTGTTATGTTTCAAGAGAACA

TAACAGTCAGATGCTTTTTTG-3'. Cells ( $1 \times 10^{5}$ cells/well) were seeded in 24-well plates and $500 \mu \mathrm{l}$ of keratinocyte-SFM medium (Invitrogen; Thermo Fisher Scientific, Inc.) was added to each well. When the cells reached $40-60 \%$ confluence, the aforementioned vectors were transfected into cells at a final concentration of $50 \mathrm{nM}$ using Lipofectamine ${ }^{\circledR} 2000$ reagent (Invitrogen; Thermo Fisher Scientific, Inc.) at $37^{\circ} \mathrm{C}$ and $5 \%$ $\mathrm{CO}_{2}$ for $48 \mathrm{~h}$, according to the manufacturer's protocol.

$R N A$ extraction and reverse transcription-quantitative $P C R$ $(R T-q P C R)$. Total RNA was extracted from adenoid tissue tissues and NP69 cells using TRIzol ${ }^{\circledR}$ reagent (Invitrogen; Thermo Fisher Scientific, Inc.) and synthesized into first-strand cDNA using an M-MLV Reverse Transcriptase kit (cat. no. 28025013; Invitrogen; Thermo Fisher Scientific, Inc.) as previously described (25). qPCR was performed using a One Step TB Green PrimeScript RT-PCR kit II (cat. no. RR086A; Takara Bio, Inc.). mRNA expression levels were normalized to U6 and GAPDH using the $2^{-\triangle \Delta C q}$ method (26). The following thermocycling conditions were used: Initial denaturation at $95^{\circ} \mathrm{C}$ for $3 \mathrm{~min}$, followed by 40 cycles at $95^{\circ} \mathrm{C}$ for $5 \mathrm{sec}, 60^{\circ} \mathrm{C}$ for $30 \mathrm{sec}$ and at $72^{\circ} \mathrm{C}$ for $45 \mathrm{sec}$, before final extension at $72^{\circ} \mathrm{C}$ for 3 min. Primer sequences are listed in Table I.

Western blotting. Cells were lysed with lysis buffer containing protease inhibitors $(50 \mathrm{mM}$ Tris- $\mathrm{HCl} \mathrm{pH} 8 ; 50 \mathrm{mM} \mathrm{NaCl}$; $0.5 \%$ NP-40). Total protein was extracted from adenoid tissues and NP69 cells using RIPA buffer (Thermo Fisher Scientific, Inc.). Protein concentration was determined using a bicinchoninic acid assay. Equal amounts of protein $(5 \mu \mathrm{g}$ for each sample) were resolved by 10\% SDS-PAGE gels and transferred to PVDF membranes. Following blocking with 5\% non-fat milk at $25^{\circ} \mathrm{C}$ for $1 \mathrm{~h}$, the membranes were immunoblotted with primary antibodies against GR $\alpha$ (cat. no. ab3580), IL-8 (cat. no. ab18672), tumor necrosis factor $\alpha$ (TNF $\alpha$; 
Table I. Primers used for reverse transcription-quantitative PCR.

\begin{tabular}{lll}
\hline Gene & \multicolumn{1}{c}{ Forward } & \multicolumn{1}{c}{ Reverse } \\
\hline$G R \alpha$ & CGAGCTCGTGTCTGTGACG & AGAAAGTCTCCCATTGCCCA \\
$I L-8$ & GAAGAGAGCTCTGTCTGGACC & ACTGGCATCTTCACTGATTCT \\
$I L-6$ & CTGGGGCCTACAGCTTTGAT & GGCCTAAGGTCCACTTGTGT \\
$I L-1 \beta$ & TATGAACTCCTTCTCCACAAGCG & AATCTTCTCCTGGGGGTACTGG \\
$X I S T$ & GCAGAAGTACCTGAGCTCGC & CTTGCTGTAGTGGTGGTCGG \\
$G A P D H$ & TGCATTTCCTTTCTGCCTCT & TGCCCACATATGCAAAGAAA \\
\hline
\end{tabular}

GR $\alpha$, glucocorticoid receptor $\alpha$; IL, interleukin; TNF $\alpha$, tumor necrosis factor $\alpha$; XIST, X inactivate-specific transcript.

cat. no. ab1793), IL-6 (cat. no. ab6672), IL-1 $\beta$ (cat. no. ab9722), Rel-B (cat. no. ab180127), Rel-C (cat. no. ab133251), P52 (cat. no. ab264236), P50 (cat. no. ab32360) and P65 (cat. no. ab16502) at a dilution of $1: 1,000$ overnight at $4{ }^{\circ} \mathrm{C}$. All primary antibodies were purchased from Abcam. After washed using TBS $+0.1 \%$ Tween for 10 min three times, the membranes were exposed to horseradish peroxidase-conjugated goat anti-rabbit antibodies (1:5,000; cat. no. sc-2357, Santa Cruz Biotechnology, Inc.) Secondary antibodies and signals were detected using an ECL detection system (Thermo Fisher Scientific, Inc.). GAPDH (cat. no. ab8245; 1:1,000; Abcam) was used as the loading control. Images were analyzed using ImageJ software (version 1.51; National Institutes of Health).

ELISA. NP69 cell supernatants cultured in 24-well plates were collected and the production of inflammatory cytokines was measured using ELISA kits (R\&D Systems, Inc.) including interleukin 6 (IL-6; cat. no. D6050), interleukin 8 (IL-8; cat. no. D8000C), tumor necrosis factor- $\alpha$ (TNF- $\alpha$; cat. no. MTA00B) and interleukin-1 $\beta$ (IL-1 $\beta$; cat. no. MLB00C), according to the manufacturer's protocols. Levels of inflammatory cytokines were quantified by normalizing the protein concentrations (27).

Bioinformatics analysis. The binding site of XIST and GRowas predicted by starBase 2.0 website (http://starbase. sysu.edu.cn).

RNA pull-down assay. RNA pull-down assays were used to confirm the interaction between XIST and GR $\alpha$. Bio-NC, Bio-XIST and Bio-XIST-Mutant constructs were synthesized by Shanghai Genepharma Co., Ltd. The sequences were shown in Supplementary Table I. XIST was labeled with biotin to generate the constructs. Sequences for the constructs are provided in Table SI. Bio-NC was used as the negative control and input, which is the cell lysate, was used as the positive control. Following this, RIPA lysis buffer (Sangon Biotech Co., Ltd.) containing RNase inhibitors (Invitrogen; Thermo Fisher Scientific, Inc.) was utilized to lyse cells. Bio-XIST-WT, Bio-XIST-MUT or Bio-NC constructs (200 pmol) were added to supernatants and Dynabeads ${ }^{\mathrm{TM}} \mathrm{M}-270$ Streptavidin (1 mg; cat. no. 65305; Invitrogen; Thermo Fisher Scientific, Inc.) and proteinase K (Sigma-Aldrich; Merck KGaA) were added and incubated with the supernatants overnight at $4^{\circ} \mathrm{C}$ to isolate the RNA. Beads were isolated from the supernatant after centrifugation $\left(2,500 \mathrm{x} \mathrm{g}, 5 \mathrm{~min}, 4^{\circ} \mathrm{C}\right)$ and washed with wash buffer (10 mM Tris- $\mathrm{HCl} \mathrm{pH} 7.5,1 \mathrm{mM}$ EDTA, $2 \mathrm{M}$ $\mathrm{NaCl}$ and $0.1 \%$ Tween-20) followed by another centrifugation step $\left(2,500 \mathrm{x} \mathrm{g}, 5 \mathrm{~min}, 4^{\circ} \mathrm{C}\right)$. The pellet was then collected and the RNA-RNA complexes were eluted using Tris-EDTA buffer (Invitrogen; Thermo Fisher Scientific, Inc.) and purified using ethanol. Subsequently, the degree of XIST and GR $\alpha$ enrichment was analyzed by RT-qPCR as aforementioned.

Statistical analysis. Data are presented as mean \pm standard deviation. For comparison between two groups, unpaired Student's t-test was applied. For comparison between multiple groups, data were analyzed by one-way ANOVA followed by Tukey's post hoc test. Pearson's correlation analysis was used to examine the correlation between the levels of XIST and GR $\alpha$. Statistical analysis was performed using SPSS software (version 22.0; IBM Corp.). $\mathrm{P}<0.05$ was considered to indicate a statistically significant difference.

\section{Results}

GR $\alpha$ is decreased in the adenoids of patients with OSAHS. Previous studies have demonstrated that GR $\alpha$ is an important factor in the inhibition of inflammation. The current study verified GR $\alpha$ expression in the adenoids of patients with OSAHS. GR $\alpha$ mRNA was significantly decreased in the adenoids of patients with OSAHS compared with healthy controls (Fig. 1A). Additionally, GR $\alpha$ protein levels were significantly decreased in the adenoids of patients with OSAHS compared with healthy controls (Fig. 1B and C). In contrast, the western blot results demonstrated that the protein levels of inflammatory cytokines, including IL-8, TNF $\alpha$, IL-6 and IL-1 $\beta$, were significantly increased in the adenoids of patients with OSAHS patients compared with healthy controls (Fig. 1D and E). In summary, GR $\alpha$ expression was decreased and inflammatory cytokine expression was increased in the adenoids of patients with OSAHS.

XIST negatively regulates GR $\alpha$ expression. A bioinformatics assay using the Starbase database demonstrated that lncRNA XIST was closely associated with GR $\alpha$. To determine whether XIST interacted with GR $\alpha$, the association between 


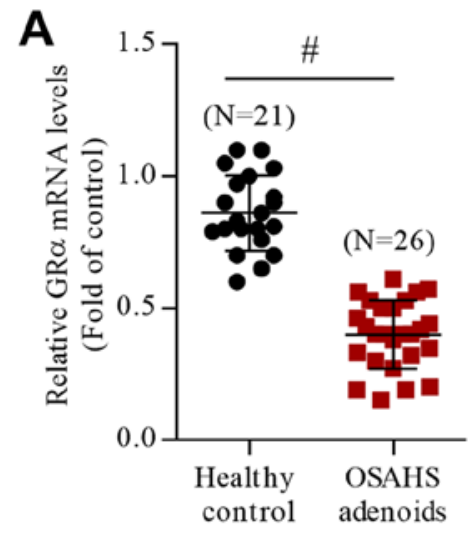

B

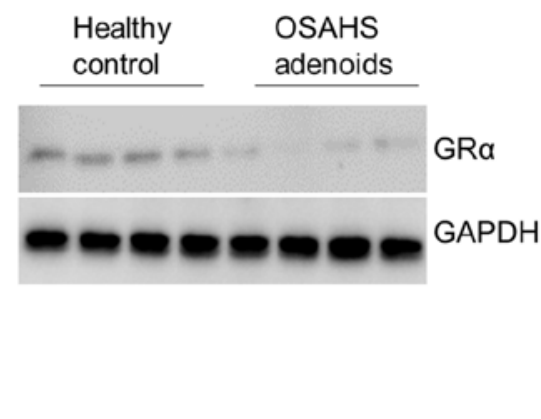

E

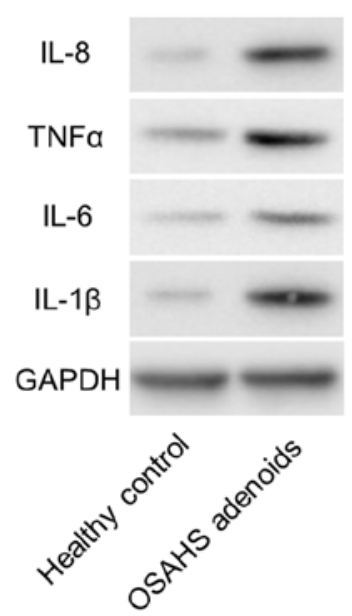

D
C

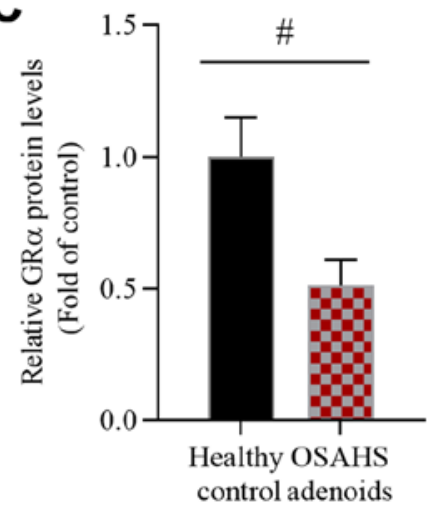

Figure 1. GR $\alpha$ is highly expressed in the adenoids of patients with OSAHS. (A) Reverse transcription-quantitative PCR data demonstrated changes in GR $\alpha$ mRNA levels. (B) Representative western blot images and (C) analyzed data reported changes in GR $\alpha$ protein levels. (D) Representative western blot images and (E) analyzed data revealed changes in the levels of inflammatory cytokines, including IL-8, TNF $\alpha$, IL-6 and IL-1 $\beta$. For IL-8, IL-6 and IL-1 $\beta$, $n=4 /$ group. For TNF $\alpha, n=5$ /group. ${ }^{\#} \mathrm{P}<0.05$ vs. the healthy control group. GR $\alpha$, glucocorticoid receptor $\alpha$; OSAHS, obstructive sleep apnea/hypopnea syndrome; IL, interleukin; TNF $\alpha$, tumor necrosis factor $\alpha$.

the expression levels of XIST and GR $\alpha$ was analyzed using Pearson's correlation analysis. According to the results, the expression of XIST was negatively correlated with the expression of GR $\alpha$ (Fig. 2A). In contrast to the expression of GR $\alpha$, RT-qPCR demonstrated that the expression of XIST was significantly increased in the adenoids of patients with OSAHS compared with healthy controls (Fig. 2B). Furthermore, pcDNA-XIST significantly increased XIST levels and XIST shRNA significantly decreased XIST levels in NP69 cells compared with those in the pcDNA and sh-NC groups, respectively (Fig. 2C). Additionally, the potential effect of XIST on GR $\alpha$ expression was investigated. XIST overexpression significantly decreased GR $\alpha$ mRNA levels and XIST shRNA significantly increased GR $\alpha$ mRNA levels compared with those in the pcDNA and sh-NC groups, respectively (Fig. 2D). Similarly, pcDNA-XIST significantly decreased GR $\alpha$ protein levels and XIST shRNA significantly increased the GR $\alpha$ protein levels compared with those in the pcDNA and sh-NC groups, respectively (Fig. 2E and F). Following this, the interaction between XIST and GR $\alpha$ was examined. The predicted binding sites for XIST and GR $\alpha$ were obtained from the Starbase database (Fig. 2G). Bio-XIST-WT and Bio-XIST-MUT constructs were generated for RNA pull-down assays. The results demonstrated that XIST and GR $\alpha$ were enriched in the Bio-XIST-WT group compared with the Bio-XIST-MUT group (Fig. 2H). These results indicated that XIST negatively regulated GR $\alpha$.

The XIST-GR $\alpha$ signaling pathway regulates the production of inflammatory cytokines. Whether the XIST-GR $\alpha$ axis regulated inflammation was investigated. GR $\alpha$ expression was significantly overexpressed by pcDNA-GR $\alpha$ transfection compared with that following pcDNA transfection (Fig. 3A). XIST significantly increased the mRNA levels (Fig. 3B), protein levels (Fig. 3C and D and E) concentrations of inflammatory cytokines, including IL- 8 , TNF $\alpha$, IL-6 and IL-1 $\beta$, compared with those in the pcDNA group. However, these stimulatory effects were reversed by XIST-MUT. Therefore, GR $\alpha$ overexpression significantly decreased the mRNA levels, protein levels and concentrations of IL-8, TNF $\alpha$, IL-6 and IL-1 $\beta$ compared with those in the pcDNA group. Furthermore, the stimulatory effect of XIST on the production of inflammatory cytokines was significantly reversed by GR $\alpha$ compared with that in the pcDNA and pcDNA-XIST + pcDNA-GR $\alpha$ 

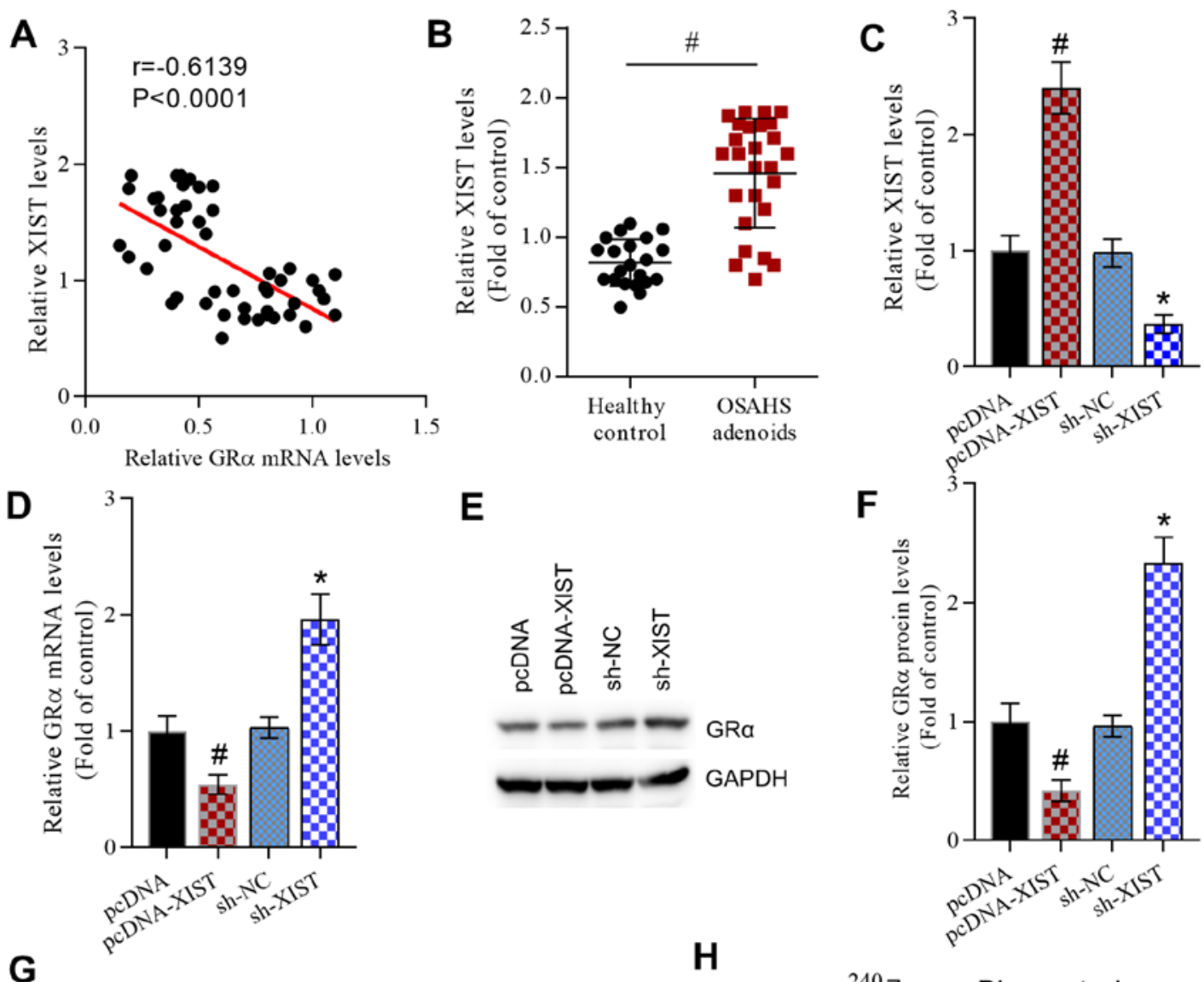

E

$\mathbf{F}$
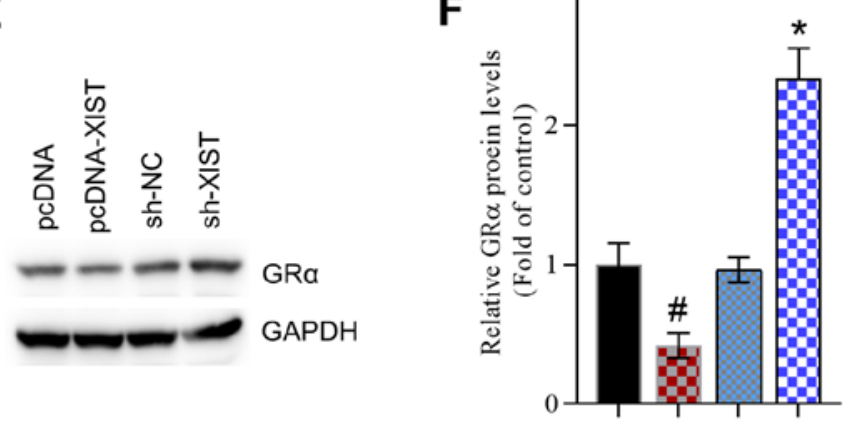

Interactions between GR $\alpha$ and XIST (Starbase):

GRa mRNA: 5' AACGUG-AACUA-ACAG-AU 3'

XIST-WT: $\quad$ 3' UUGGACAUUAAUGUG-CAUA 5'

XIST-MUT: 5' AACGUGAAAAUAGAC-GAAU 3'

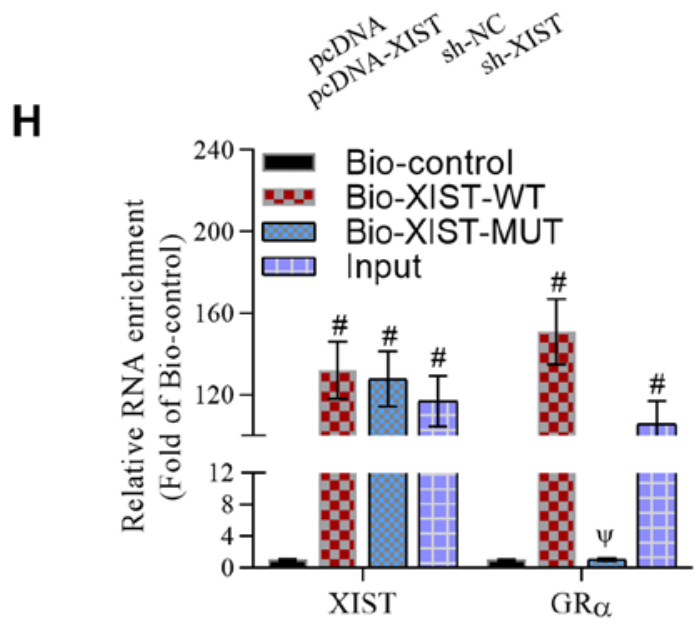

Figure 2. XIST negatively regulates GR $\alpha$ expression. (A) Pearson's correlation analysis indicated a negative correlation between the XIST and GR $\alpha$ mRNA levels. $r=-0.6139$. $P<0.0001$. (B) RT-qPCR revealed increased XIST expression levels in the adenoids of patients with OSAHS compared with healthy controls. ${ }^{\#} \mathrm{P}<0.05$ as indicated. (C) RT-qPCR data demonstrated the effect of the overexpression or knockdown of XIST on XIST levels in NP69 cells. ${ }^{\#} \mathrm{P}<0.05$ vs. the pcDNA group and ${ }^{*} \mathrm{P}<0.05$ vs. the sh-NC group. (D) RT-qPCR data revealed the effect of the overexpression or knockdown of XIST on GR $\alpha$ mRA levels in NP69 cells. "P<0.05 vs. the pcDNA group and " $\mathrm{P}<0.05$ vs. the sh-NC group. (E) Representative western blot images and (F) analyzed data demonstrated the effect of the overexpression or knockdown of XIST on GR $\alpha$ protein levels in NP69 cells. ${ }^{~} \mathrm{P}<0.05$ vs. the pcDNA group and ${ }^{*} \mathrm{P}<0.05$ vs. the sh-NC group. (G) The binding sites and mutated sites of XIST and GR $\alpha$. (H) RNA pull-down assays confirmed the interaction between XIST and GR $\alpha$. " $\mathrm{P}<0.05$ vs. the Bio-control group and ${ }^{\Psi} \mathrm{P}<0.05$ vs. the Bio-XIST-WT group. No significance between GR $\alpha$ enrichment in Bio-XIST-MUT and Bio-control group. For XIST, $\mathrm{n}=4$ /group. For GR $\alpha, \mathrm{n}=5$ /group. XIST, X inactivate-specific transcript; GR $\alpha$, glucocorticoid receptor $\alpha$; RT-qPCR, reverse transcription-quantitative PCR; OSAHS, obstructive sleep apnea/hypopnea syndrome; sh, short hairpin; NC, negative control; WT, wild-type; MUT, mutant.

groups (Fig. 3F). These data indicated that the XIST-GR $\alpha$ signaling pathway contributed to the regulation of inflammatory cytokine production.

$X I S T$ regulates adenoidal inflammation through the $G R \alpha$-mediated $N F-\kappa B$ pathway. Finally, we evaluated whether $\mathrm{NF}-\kappa \mathrm{B}$ was involved in XIST-GR $\alpha$-induced inflammation. XIST significantly increased the protein levels of Rel-B, c-Rel, P52, P50 and P65 compared with that in the pcDNA/DMSO group (Fig. 4A and B). These effects were significantly inhibited by the NF- $\mathrm{B}$ inhibitor EVP4593. Additionally, GR $\alpha$ inactivated the $N F-\kappa B$ pathway and significantly reversed XIST-induced expression of $N F-\kappa B$ pathway-associated proteins compared with that in the pcDNA-XIST/DMSO group. Similarly, XIST induced a significant increase in the mRNA levels (Fig. 4C) and concentrations (Fig. 4D) of IL-8, TNF $\alpha$, IL- 6 and IL-1 $\beta$ compared with those in the pcDNA/DMSO group. Furthermore, compared with those in the pcDNA-XIST/DMSO group, these levels were significantly inhibited by EVP4593 or GR $\alpha$, counteracting the effects of 


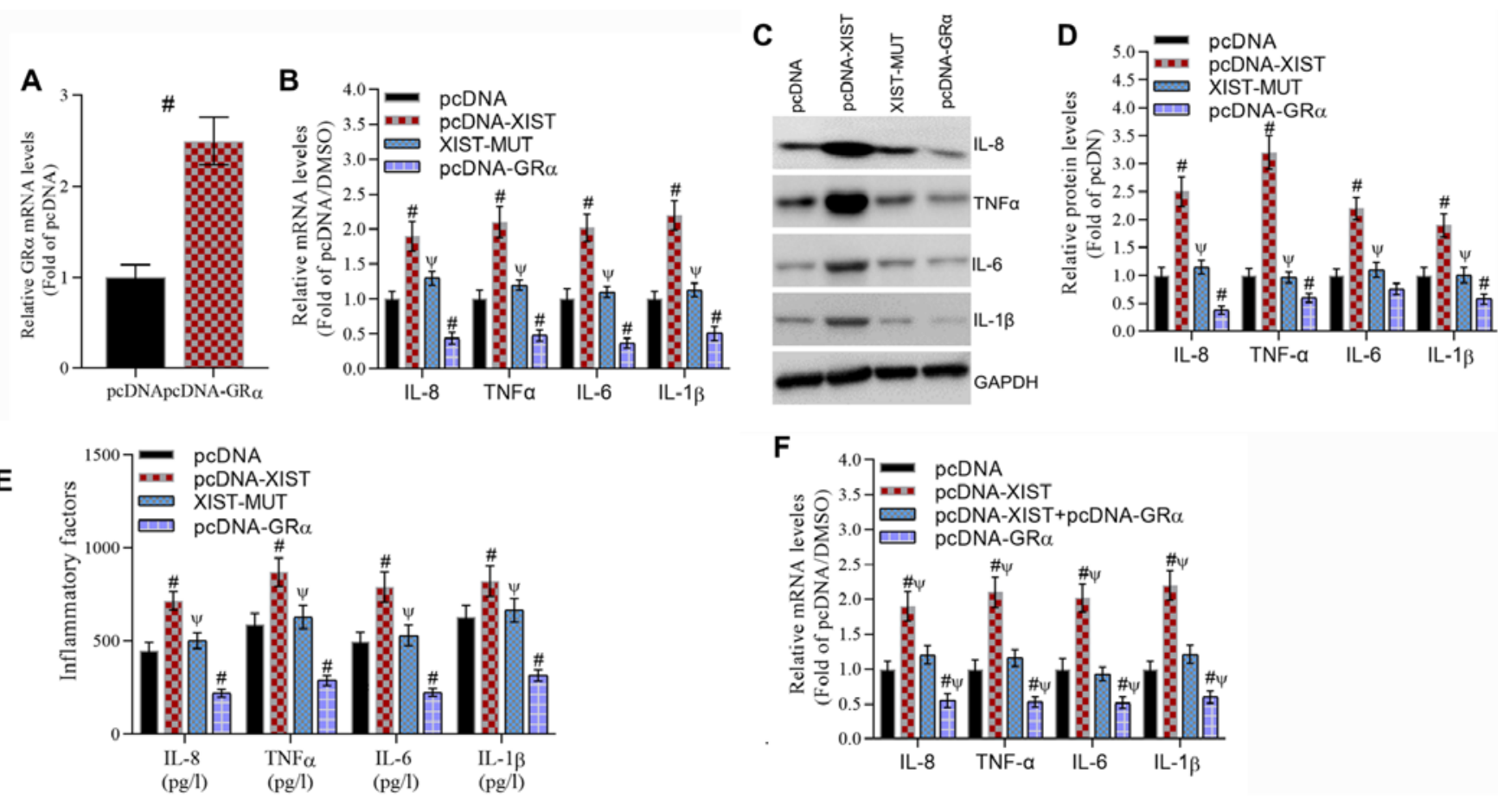

Figure 3. XIST promotes the production of inflammatory cytokines, including IL-8, TNF $\alpha$, IL-6 and IL-1 $\beta$, that depend on the binding of XIST and GR $\alpha$. (A) RT-qPCR data demonstrated the effect of GR $\alpha$ overexpression on GR $\alpha$ mRNA levels. ${ }^{\#} \mathrm{P}<0.05$ vs. the pcDNA group. (B) RT-qPCR data revealed the effect of XIST-WT, XIST-MUT and GR $\alpha$ overexpression on the mRNA levels of inflammatory cytokines. ${ }^{*} \mathrm{P}<0.05$ vs. the pcDNA group and ${ }^{\Psi} \mathrm{P}<0.05$ vs. the XIST group. (C) Representative western blotting images and (D) analyzed data demonstrated the effect of XIST-WT, XIST-MUT and GR $\alpha$ overexpression on the protein levels of inflammatory cytokines. ${ }^{~} \mathrm{P}<0.05$ vs. the pcDNA group and ${ }^{\Psi} \mathrm{P}<0.05$ vs. the XIST group. (E) Analyzed data revealed the effect of XIST-WT, XIST-MUT and GR $\alpha$ overexpression on the protein levels of inflammatory cytokines as determined by ELISA. ${ }^{*} \mathrm{P}<0.05$ vs. the pcDNA group and ${ }^{\Psi} \mathrm{P}<0.05$ vs. the XIST group. (F) Analyzed data demonstrated that the inhibitory effect of GR $\alpha$ overexpression reversed the stimulatory effect of XIST on the protein levels of inflammatory cytokines as determined by ELISA. " $\mathrm{P}<0.05$ vs. the pcDNA group and ${ }^{\Psi} \mathrm{P}<0.05$ vs. the XIST+GR $\alpha$ group. For IL- 8 , IL-6 and IL- $1 \beta$, $\mathrm{n}=5$ /group. For TNF $\alpha, \mathrm{n}=6$ /group. XIST, $\mathrm{X}$ inactivate-specific transcript; GR $\alpha$, glucocorticoid receptor $\alpha$; RT-qPCR, reverse transcription-quantitative PCR; WT, wild-type; MUT, mutant; IL, interleukin; TNF $\alpha$, tumor necrosis factor $\alpha$.

XIST in facilitating the inflammatory response. These results indicated that XIST induced an inflammatory response through the GR $\alpha$-mediated NF- $\mathrm{B}$ pathway.

\section{Discussion}

OSAHS is a risk factor for hypertension, arrhythmia, coronary heart disease and other diseases (28). Therefore, investigating the specific molecular regulatory mechanisms of OSAHS is crucial. The current study evaluated whether GR $\alpha$ was involved in the inflammatory response in OSAHS. The results demonstrated that GR $\alpha$ expression was significantly decreased and XIST expression was significantly increased in the adenoids of patients with OSAHS compared with healthy controls. Further research revealed that XIST interacted with GR $\alpha$ and decreased GR $\alpha$ expression. Functionally, XIST increased the production of inflammatory cytokines, including IL-8, TNF- $\alpha$, IL- 6 and IL-1 $\beta$, while GR $\alpha$ decreased their production. Furthermore, the results demonstrated that XIST-GR $\alpha$ axis-mediated inflammation was significantly inhibited by the NF- $\kappa \mathrm{B}$ inhibitor EVP4593. These results indicated that the XIST-GR $\alpha-\mathrm{NF}-\kappa \mathrm{B}$ signaling pathway contributed to inflammation in the adenoids of patients with OSAHS.

The current study reported that GR $\alpha$ was decreased in patients with OSAHS, which was consistent with a previous study that revealed that GR $\alpha$ was decreased in the tonsils of children with OSAHS (10). Furthermore, the present study demonstrated that decreased GR $\alpha$ contributed to increased inflammation in patients with OSAHS. Furthermore, the present study reported that XIST was upregulated and decreased the expression of GR $\alpha$ in patients with OSAHS. Consistent with the present study, accumulating data have demonstrated that XIST is upregulated and involved in various diseases, including basal-like human breast cancer (15) and cystic fibrosis (16). Shenoda et al (29) reported that XIST promotes inflammation through the pro-inflammatory transcription factor yin yang 1 in patients with complex regional pain syndrome. Those studies indicated that XIST served a critical role in inflammation. Additionally, XIST promotes the progression of non-small cell lung cancer through the microRNA (miR)-335/superoxide dismutase 2/reactive oxygen species pathway (30). Inhibition of XIST suppresses cell proliferation via modulation of the miR-744/ring finger protein 1 axis in non-small cell lung cancer (31). Suppression of XIST increases the secretion of exosomal miR-503, which triggers M1-M2 polarization of microglia and promotes brain metastasis (32). The results of the current study indicated that XIST interacted with GR $\alpha$ and inhibited GR $\alpha$ expression. GR $\alpha$ has been previously reported to serve an anti-inflammatory role in cardiomyocytes (33). The present study revealed that GR $\alpha$ alleviated the inflammatory response and inactivated the $\mathrm{NF}-\kappa \mathrm{B}$ pathway in NP69 cells.

Numerous previous studies have demonstrated that NF- $\kappa \mathrm{B}$ serves a vital role in inflammation. For instance, it has been 
A

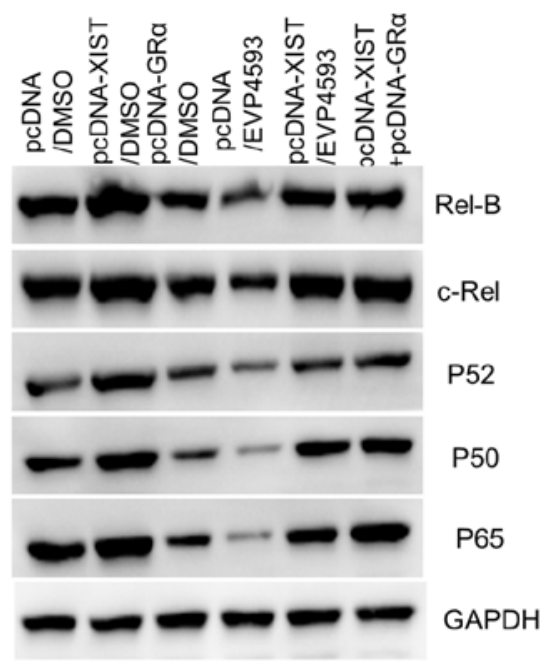

B

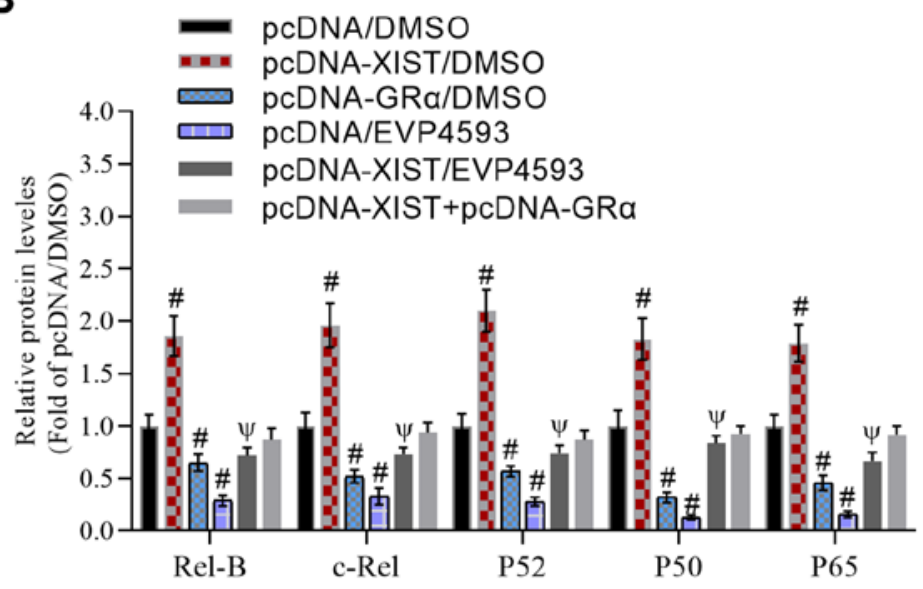

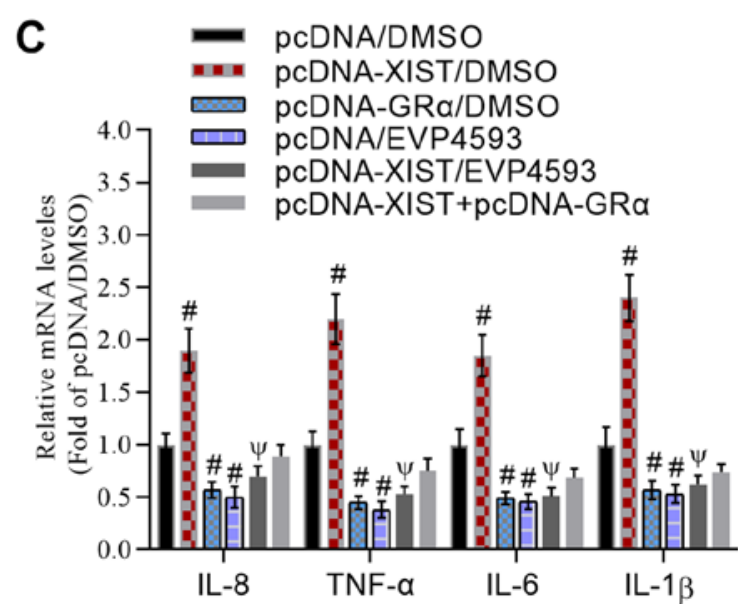

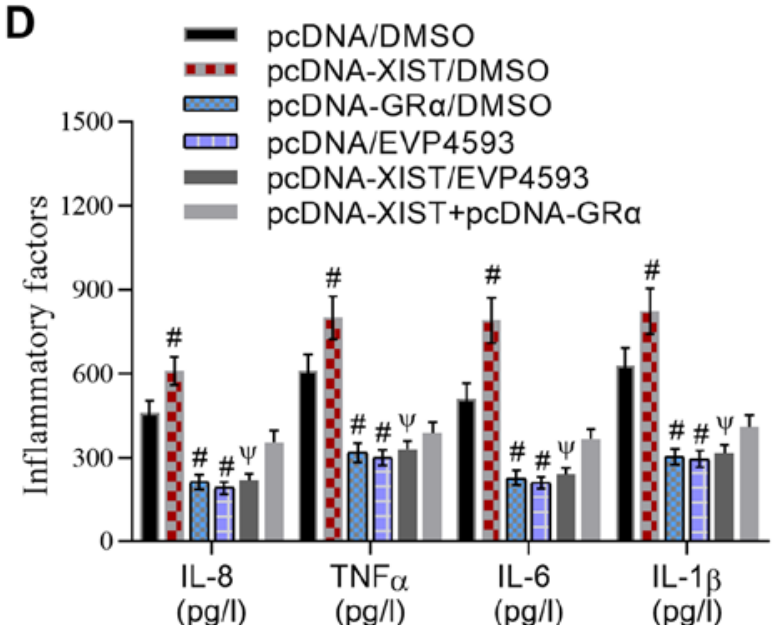

Figure 4. XIST regulates adenoidal inflammation through the GR $\alpha$-mediated NF- $\kappa$ B pathway. (A) Representative western blot images and (B) analyzed data demonstrated the inhibitory effect of the NFKB inhibitor EVP4593 and GR $\alpha$ on the XIST-induced increase in protein levels of Rel-B, c-Rel, P52, P50 and P65. (C) Reverse transcription-quantitative PCR data revealed the inhibitory effect of EVP4593 and GR $\alpha$ on the XIST-induced increase in the mRNA levels of inflammatory cytokines. (D) Analyzed data reported the inhibitory effect of EVP4593 and GR $\alpha$ on the XIST-induced increase in the protein levels of inflammatory cytokines as determined by ELISA. For IL-8, IL- 6 and IL-1 $\beta, n=5 /$ group. For TNF $\alpha, n=6 /$ group. ${ }^{"} \mathrm{P}<0.05$ vs. the pcDNA/DMSO group and ${ }^{\Psi} \mathrm{P}<0.05$ vs . the XIST/DMSO group. XIST, X inactivate-specific transcript; GR $\alpha$, glucocorticoid receptor $\alpha$; NF- $\kappa \mathrm{B}$, nuclear factor $\kappa$-light-chain-enhancer of activated B cells; IL, interleukin; TNF $\alpha$, tumor necrosis factor $\alpha$.

reported that $\mathrm{NF}-\kappa \mathrm{B}$ upregulated the synthesis and secretion of various pro-inflammatory cytokines, including TNF- $\alpha$, IL-1 $\beta$ and IL-6. Fu et al (34) reported that pharmacologically inhibiting neddylation with MLN4924 suppressed pro-inflammatory cytokine generation through the NF- $\kappa \mathrm{B}$ pathway in lipopolysaccharide (LPS)-stimulated HK2 cells and attenuates renal inflammation in LPS-induced acute kidney damage. Zhao et al (35) revealed that XIST upregulated TNF- $\alpha$ induced protein 1 expression to increase NF-kB activity and, therefore, exacerbating neuropathic pain in a rat model. XIST alleviates LPS-induced cell injury by modulating the Janus kinase/signal transducer and activator of transcription and $\mathrm{NF}-\kappa \mathrm{B}$ pathways (36). However, Ma et al (17) demonstrated that the expression of XIST was promoted by the activation of the NF- $\kappa \mathrm{B}$ pathway and that XIST generated a negative feedback loop to regulate the NF- $\kappa \mathrm{B} / \mathrm{NLR}$ family CARD domain containing 6, leucine-rich repeat and PYD domain-containing protein 3 inflammasome pathways to mediate inflammatory processes. The aforementioned studies indicated that the association between XIST and NF- $\kappa \mathrm{B}$ is complex. The present study identified that GR $\alpha$ suppressed activation of the NF- $\kappa \mathrm{B}$ pathway and that XIST activated the NF- $\kappa$ B pathway through the inhibition of GR $\alpha$. However, the underlying mechanism by which $\mathrm{GR} \alpha$ inactivates the $\mathrm{NF}-\kappa \mathrm{B}$ pathway remains unclear. Future studies will investigate the mechanism by which $\mathrm{GR} \alpha$ suppresses the activation of the $\mathrm{NF}-\kappa \mathrm{B}$ pathway in the progression of OSAHS.

In summary, the present study demonstrated that XIST decreased the expression of GR $\alpha$, thereby contributing to the initiation and development of OSAHS through an $\mathrm{NF}-\kappa \mathrm{B}$-dependent signaling pathway. This may provide a potential target for the treatment of OSAHS. 


\section{Acknowledgements}

Not applicable.

\section{Funding}

The current study was supported by the Hangzhou Science and Technology Development Plan Project (grant nos. 20170533B94 and 20170533B27).

\section{Availability of data and materials}

The datasets used and/or analyzed during the current study are available from the corresponding author on reasonable request.

\section{Author's contributions}

ZZ conceived and designed the experiments. ZZ, HN, YL and BJ performed the experiments. $\mathrm{ZZ}$ and $\mathrm{HN}$ analyzed the data. $\mathrm{ZZ}$ and HN can authenticate the raw data in this study. All authors agreed to be accountable for all aspects of the work. All authors read and approved the final manuscript.

\section{Ethics approval and consent to participate}

The current study was approved by the Ethics Committee and Animal Care Committee of the Affiliated Hangzhou First People's Hospital, Zhejiang University School of Medicine (Hangzhou, China). Informed consent was obtained from all participants included in the current study. All procedures performed in studies involving human participants were in accordance with the ethical standards of the institutional and/or national research committee and with the 1964 Declaration of Helsinki and its later amendments or comparable ethical standards.

\section{Patient consent for publication}

Not applicable.

\section{Competing interests}

The authors declare that they have no competing interests.

\section{References}

1. Zhang L, Ou X, Zhu T and Lv X: Beneficial effects of estrogens in obstructive sleep apnea hypopnea syndrome. Sleep Breath 24: $7-13,2020$

2. Gonzaga C, Bertolami A, Bertolami M, Amodeo C and Calhoun D: Obstructive sleep apnea, hypertension and cardiovascular diseases. J Hum Hypertens 29: 705-12, 2015.

3. Jordan AS and McEvoy RD: Gender differences in sleep apnea: Epidemiology, clinical presentation and pathogenic mechanisms. Sleep Med Rev 7: 377-389, 2003.

4. Morong S, Hermsen B and de Vries N: Sleep-disordered breathing in pregnancy: A review of the physiology and potential role for positional therapy. Sleep Breath 18: 31-37, 2014.

5. Snyder B and Cunningham RL: Sex differences in sleep apnea and comorbid neurodegenerative diseases. Steroids 133: 28-33, 2018.

6. Evans RM: The steroid and thyroid hormone receptor superfamily. Science 240: 889-895, 1988.
7. Ren R, Oakley RH, Cruz-Topete D and Cidlowski JA: Dual role for glucocorticoids in cardiomyocyte hypertrophy and apoptosis. Endocrinology 153: 5346-5360, 2012.

8. Kim HK, Jeong YJ, Song IS, Noh YH, Seo KW, Kim M and Han J: Glucocorticoid receptor positively regulates transcription of FNDC5 in the liver. Sci Rep 7: 43296, 2017.

9. Kuo T, Chen TC, Yan S, Foo F, Ching C, McQueen A and Wang JC: Repression of glucocorticoid-stimulated angiopoietin-like 4 gene transcription by insulin. J Lipid Res 55: 919-28, 2014.

10. Chen X and Li JR: Glucocorticoid receptor expression in the tonsils of children with obstructive sleep apnea hypopnea syndrome. Genet Mol Res 15: 2016.

11. Choi SW, Kim HW and Nam JW: The small peptide world in long noncoding RNAs. Brief Bioinform 20: 1853-1864, 2019.

12. Barth DA, Slaby O, Klec C, Juracek J, Drula R, Calin GA and Pichler M: Current concepts of non-coding RNAs in the pathogenesis of non-clear cell renal cell carcinoma. Cancers (Basel) 11: 1580, 2019.

13. Rapicavoli NA, Qu K, Zhang J, Mikhail M, Laberge RM and Chang HY: A mammalian pseudogene lncRNA at the interface of inflammation and anti-inflammatory therapeutics. Elife 2: $\mathrm{e} 00762,2013$.

14. Carpenter S, Aiello D, Atianand MK, Ricci EP, Gandhi P, Hall LL, Byron M, Monks B, Henry-Bezy M, Lawrence JB, et al: A long noncoding RNA mediates both activation and repression of immune response genes. Science 341: 789-792, 2013.

15. Richardson AL, Wang ZC, De Nicolo A, Lu X, Brown M, Miron A, Liao X, Iglehart JD, Livingston DM and Ganesan S: X chromosomal abnormalities in basal-like human breast cancer. Cancer Cell 9: 121-132, 2006.

16. McKiernan PJ, Molloy K, Cryan SA, McElvaney NG and Greene CM: Long noncoding RNA are aberrantly expressed in vivo in the cystic fibrosis bronchial epithelium. Int $\mathrm{J}$ Biochem Cell Biol 52: 184-191, 2014.

17. Ma M, Pei Y, Wang X, Feng J, Zhang Y and Gao MQ: LncRNA XIST mediates bovine mammary epithelial cell inflammatory response via NF-kappaB/NLRP3 inflammasome pathway. Cell Prolif 52: e12525, 2019

18. Sadeghi A, Rostamirad A, Seyyedebrahimi S and Meshkani R: Curcumin ameliorates palmitate-induced inflammation in skeletal muscle cells by regulating JNK/NF-kB pathway and ROS production. Inflammopharmacology 26: 1265-1272, 2018.

19. Nedjai B, Hitman GA, Church LD, Minden K, Whiteford ML, McKee S, Stjernberg S, Pettersson T, Ranki A, Hawkins PN, et al: Differential cytokine secretion results from p65 and c-Rel $\mathrm{NF}-\kappa \mathrm{B}$ subunit signaling in peripheral blood mononuclear cells of TNF receptor-associated periodic syndrome patients. Cell Immunol 268: 55-59, 2011.

20. Ruland J: Return to homeostasis: Downregulation of NF- $\kappa \mathrm{B}$ responses. Nature Immunol 12: 709-714, 2011.

21. Lai JL, Liu YH, Liu C, Qi MP, Liu RN, Zhu XF, Zhou QG, Chen YY, Guo AZ and Hu CM: Indirubin inhibits LPS-induced inflammation via TLR4 abrogation mediated by the NF- $\kappa \mathrm{B}$ and MAPK signaling pathways. Inflammation 40: 1-12, 2017.

22. Yancy CW, Jessup M, Bozkurt B, Butler J, Casey DE Jr, Drazner MH, Fonarow GC, Geraci SA, Horwich T, Januzzi JL, et al: 2013 ACCF/AHA guideline for the management of heart failure: A report of the American College of Cardiology Foundation/American Heart Association Task Force on Practice Guidelines. J Am Coll Cardiol 62: 147-239, 2013.

23. Ye XH, Chen H, Yu Q and Zhu QL: Liver X receptor gene expression is enhanced in children with obstructive sleep apnea-hyperpnoea syndrome and cyclooxygenase-2 (COX-2) is correlated with severity of obstructive sleep apnea-hypopnea syndrome (OSAHS). Med Sci Monit 23: 3261-3268, 2017.

24. Guo Z, Wang Y, Yang J, Zhong J, Liu X and Xu M: KAI1 overexpression promotes apoptosis and inhibits proliferation, cell cycle, migration, and invasion in nasopharyngeal carcinoma cells. Am J Otolaryngol 38: 511-517, 2017.

25. Pathare AD and Deshpande AS: HGV-HCV/HBV co-infection in India: A pilot study. Asian J Transfus Sci 7: 48-50, 2013.

26. Livak KJ and Schmittgen TD: Analysis of relative gene expression data using real-time quantitative PCR and the 2(-Delta Delta C(T)) method. Methods 25: 402-8, 2001.

27. Yu X, Wang D, Wang X, Sun S, Zhang Y, Wang S, Miao R, Xu X and Qu X: CXCL12/CXCR4 promotes inflammation-driven colorectal cancer progression through activation of RhoA signaling by sponging miR-133a-3p. J Exp Clin Cancer Res 38: 32, 2019.

28. Burman D: Sleep disorders: Sleep-related breathing disorders. FP Essent 460: 11-21, 2017. 
29. Shenoda BB, Tian Y, Alexander GM, Aradillas-Lopez E Schwartzman RJ and Ajit SK: miR-34a-mediated regulation of XIST in female cells under inflammation. J Pain Res 11: 935-945, 2018.

30. Liu J,YaoL,Zhang M,Jiang J,Yang MandWang Y:Downregulation of LncRNA-XIST inhibited development of non-small cell lung cancer by activating miR-335/SOD2/ROS signal pathway mediated pyroptotic cell death. Aging 11: 7830-7846, 2019.

31. Wang J, Cai H, Dai Z and Wang G: Down-regulation of lncRNA XIST inhibits cell proliferation via regulating miR-744/RING1 axis in non-small cell lung cancer. Clin Sci (Lond) 133: 1567-1579, 2019.

32. Xing F, Liu Y, Wu SY, Wu K, Sharma S, Mo YY, Feng J, Sanders S, Jin G, Singh R, et al: Loss of XIST in breast cancer activates MSN-c-Met and reprograms microglia via exosomal miRNA to promote brain metastasis. Cancer Res 78: 4316-4330, 2018.

33. Song YX, Ou YM and Zhou JY: Gracillin inhibits apoptosis and inflammation induced by lipopolysaccharide (LPS) to alleviate cardiac injury in mice via improving miR-29a. Biochem Biophys Res Commun 523: 580-587, 2020.
34. Fu Z, Liao W, Ma H, Wang Z, Jiang $M$, Feng $X$ and Zhang W: Inhibition of neddylation plays protective role in lipopolysaccharide-induced kidney damage through CRLmediated NF-kappaB pathways. Am J Transl Res 11: 2830-2842, 2019.

35. Zhao Y,Li S, Xia N, Shi Y and Zhao CM: Effects of XIST/miR-137 axis on neuropathic pain by targeting TNFAIP1 in a rat model. J Cell Physiol 233: 4307-4316, 2018.

36. Zhang Y,Zhu Y, Gao G and Zhou Z: Knockdown XIST alleviates LPS-induced WI-38 cell apoptosis and inflammation injury via targeting miR-370-3p/TLR4 in acute pneumonia. Cell Biochem Funct 37: 348-358, 2019.

(c) (1) (9) This work is licensed under a Creative Commons International (CC BY-NC-ND 4.0) License. 